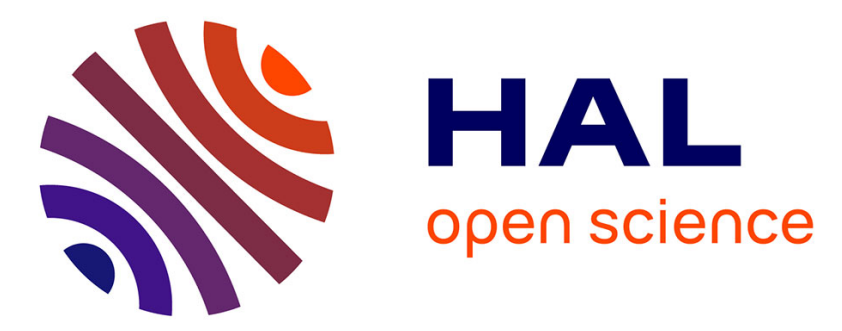

\title{
Convergence of a semi-Lagrangian scheme for the reduced Vlasov-Maxwell system for laser-plasma interaction
}

\author{
Mihai Bostan, Nicolas Crouseilles
}

\section{To cite this version:}

Mihai Bostan, Nicolas Crouseilles. Convergence of a semi-Lagrangian scheme for the reduced VlasovMaxwell system for laser-plasma interaction. Numerische Mathematik, 2009, 112, pp.169-195. hal00773420

\author{
HAL Id: hal-00773420 \\ https://hal.science/hal-00773420
}

Submitted on 14 Jan 2013

HAL is a multi-disciplinary open access archive for the deposit and dissemination of scientific research documents, whether they are published or not. The documents may come from teaching and research institutions in France or abroad, or from public or private research centers.
L'archive ouverte pluridisciplinaire HAL, est destinée au dépôt et à la diffusion de documents scientifiques de niveau recherche, publiés ou non, émanant des établissements d'enseignement et de recherche français ou étrangers, des laboratoires publics ou privés. 


\title{
Convergence of a semi-Lagrangian scheme for the reduced Vlasov-Maxwell system for laser-plasma interaction
}

\author{
Mihai Bostan ${ }^{*}$ Nicolas Crouseilles ${ }^{\dagger}$
}

(21st August 2008)

\begin{abstract}
The subject matter of this paper concerns the numerical approximation of reduced Vlasov-Maxwell models by semi-Lagrangian schemes. Such reduced systems have been introduced recently in the literature for studying the laserplasma interaction. We recall the main existence and uniqueness results on these topics, we present the semi-Lagrangian scheme and finally we establish the convergence of this scheme.
\end{abstract}

\section{Introduction}

The Vlasov-Maxwell system is a model which describes the motion of a collisionless plasma under the effects of the transport and the self-consistent forces (electro-magnetic fields). The solution to the Vlasov equation is a distribution function $f=f(t, x, p)$ depending on the time $t \in \mathbb{R}_{+}$, the space variable $x$ and the momentum $p$ of particles, whereas the self-consistent electro-magnetic fields satisfy the Maxwell equations.

The numerical resolution of the Vlasov equation is most of the time performed through Particle In Cell (PIC) methods, which consist in approximating the plasma by a finite number of macro-particles; the trajectories of these particles are computed from the characteristic curves associated to the Vlasov equation. However, the evolution of the self-consistent fields are computed using a mesh of the physical space (see [4] for more details). Even if this kind of method enables to get some satisfactory results with relatively few particles, for some applications however, it is

*Laboratoire de Mathématiques de Besançon, UMR CNRS 6623, Université de Franche-Comté, 16 route de Gray, 25030 Besançon Cedex France. E-mail : mbostan@univ-fcomte.fr

†IRMA, Université Louis Pasteur, 7 rue René Descartes, 67084 Strasbourg Cedex France. Email : crouseil@math.u-strasbg.fr 
well known that the numerical noise becomes too important. Consequently, methods relying on a discretization of the phase space have been proposed (see $[18,17])$ and seem to be very efficient when the particles in the tail of the distribution play an important role for example. Among these methods, finite volume schemes (see $[5,9,12,13])$ have been successfully implemented; even if they are known to be robust, they are quite dissipative and suffer from the fact that they are constrained by a severe CFL condition on the time step. On the other hand, the semi-Lagrangian method consists of directly computing the distribution function on a grid of the phase space, updating the unknowns by following the characteristic curves backward at each time step and approximating the value at the origin of the characteristics by an interpolation method (cubic splines, Lagrange or Hermite polynomials, ...). We refer the reader to $[19,3]$ for more details.

In this work we consider a reduced Vlasov-Maxwell system. This model describes the motion of the electrons in the laser-plasma interaction context and has been recently introduced in the literature by the physicists [15]. To derive such a model, the key points are the following: starting from the Vlasov-Maxwell equations in one dimension in space (called $x$ ) and three dimensions in momentum, we make the assumption that the motions of interest are faster along the direction of propagation of the laser than in the associated transversal directions. Then, it is reasonable to consider that the electrons are monokinetic in the directions transversal to $x$. We refer the reader to $[16,14]$ for more details.

After some computations (see [8]) one gets the system

$$
\begin{gathered}
\partial_{t} f+\frac{p}{\gamma_{1}} \partial_{x} f-\left(E(t, x)+\frac{A(t, x)}{\gamma_{2}} \partial_{x} A\right) \partial_{p} f=0 \\
\partial_{t}^{2} A-\partial_{x}^{2} A=-\rho_{\gamma_{2}}(t, x) A(t, x) \\
\partial_{t} E=j(t, x) \\
\partial_{x} E=\rho_{\text {ext }}(x)-\rho(t, x)
\end{gathered}
$$

where $\left\{\rho, \rho_{\gamma_{2}}, j\right\}(t, x)=\int_{\mathbb{R}}\left\{1, \frac{1}{\gamma_{2}}, \frac{p}{\gamma_{1}}\right\} f(t, x, p) d p, \gamma_{1}=\gamma_{2}=1$ in the non-relativistic case (NR), $\gamma_{1}=\left(1+p^{2}\right)^{1 / 2}, \gamma_{2}=1$ in the quasi-relativistic case (QR) and $\gamma_{1}=\gamma_{2}=$ $\left(1+p^{2}+A(t, x)^{2}\right)^{1 / 2}$ in the fully-relativistic case (FR). The notation $\rho_{\text {ext }}$ stands for the concentration of the ion background. We supplement these equations with the initial conditions

$$
f(0, x, p)=f_{0}(x, p),(x, p) \in \mathbb{R}^{2}, \quad\left(E, A, \partial_{t} A\right)(0, x)=\left(E_{0}, A_{0}, A_{1}\right)(x), x \in \mathbb{R} .
$$

The notation $\gamma$ means that we consider QR regimes, that is $\gamma_{1}=\gamma=\left(1+p^{2}\right)^{1 / 2}, \gamma_{2}=$ 1. This model involves the classical electric field derived from the Poisson equation, but also a nonlinear term which depends on the potential vector; this potential vector satisfies a wave type equation, with second member proportional to the electron concentration and the potential vector. 
Some previous works were dealt with these Vlasov-Maxwell models from the numerical point of view (see $[14,15])$. The theoretical study of the above models has been performed in $[8,6]$.

We are looking for solutions by characteristics, i.e., solutions with lipschitz continuous electro-magnetic field and electron density constant along the characteristics $(X, P)=(X, P)(s ; t, x, p)$ of $(1)$ defined by

$$
\frac{d X}{d s}=\frac{P(s)}{\gamma_{1}}, \frac{d P}{d s}=-E(x, X(s))-\frac{A(s, X(s))}{\gamma_{2}} \partial_{x} A(s, X(s)),
$$

with the conditions

$$
X(s=t ; t, x, p)=x, \quad P(s=t ; t, x, p)=p .
$$

The existence and uniqueness of the solution by characteristics (or mild solution) hold globally in time in the QR and FR cases, but only locally in time in the NR case.

The main goal of this work is the study of eulerian numerical schemes for these Vlasov-Maxwell models. More precisely, we focus our attention to the convergence analysis of a semi-Lagrangian scheme for approximating these models. Similar convergence studies have been achieved by several authors for the Vlasov-Poisson equations, we cite here $[11,1,2,7]$. The present work intends to adapt these results within the framework of the reduced Vlasov-Maxwell models. For the sake of simplicity we investigate here the QR case but similar methods apply for the NR and FR cases.

The paper is organized as follows. In Section 2 we recall the existence and uniqueness results for the solution by characteristics of the reduced Vlasov-Maxwell system. Some regularity results are presented as well. In Section 3 we present the semi-Lagrangian scheme applied to the QR Vlasov-Maxwell system and expose the notations to be used in the sequel. The last section is devoted to the convergence analysis. We state our main result and establish an error estimate for the semiLagrangian scheme previously introduced.

\section{Existence and uniqueness results}

In this section we recall the existence and uniqueness results for the mild solution in the QR and FR cases. Based on the results in [6] (cf. Theorem 4.2, pp. 185) for the FR case, which adapt easily to the QR case, one gets

Theorem 2.1 Assume that $f_{0}$ is non negative, $\left(1+|p|^{k}\right) f_{0}$ belongs to $L^{1}\left(\mathbb{R}^{2}\right)$ (with $k=2$ in the $Q R$ case and $k=1$ in the $F R$ case) and there is $n_{0}: \mathbb{R} \rightarrow \mathbb{R}_{+}$non 
decreasing on $\mathbb{R}_{-}$and non increasing on $\mathbb{R}_{+}$such that

$$
\begin{aligned}
(H) & f_{0}(x, p) \leq n_{0}(p), \quad(x, p) \in \mathbb{R}^{2} \\
\left(H_{k}\right) & M_{k}:=\int_{\mathbb{R}}|p|^{k} n_{0}(p) d p<+\infty \\
\left(H_{\infty}\right) & M_{\infty}:=\left\|n_{0}\right\|_{L^{\infty}(\mathbb{R})}<+\infty .
\end{aligned}
$$

We suppose also that $\rho_{\mathrm{ext}}$ is non negative, belongs to $L^{1}(\mathbb{R}) \cap L^{\infty}(\mathbb{R}), E_{0}^{\prime}=\rho_{\text {ext }}-$ $\int_{\mathbb{R}} f_{0} d p, A_{0} \in W^{2, \infty}(\mathbb{R}), A_{1} \in W^{1, \infty}(\mathbb{R})$. Then there is a unique global solution $(f, E, A)$ for the system (1-5) (in the $Q R$ and $F R$ cases) verifying

$$
\begin{gathered}
f \geq 0, \quad\left(1+|p|^{k}\right) f \in L^{\infty}\left([0, T] ; L^{1}\left(\mathbb{R}^{2}\right)\right), \quad \int_{\mathbb{R}} f(\cdot, \cdot, p)\left(1+|p|^{k}\right) d p \in L^{\infty}([0, T] \times \mathbb{R}) \\
E \in W^{1, \infty}([0, T] \times \mathbb{R}), \quad A \in W^{2, \infty}([0, T] \times \mathbb{R})
\end{gathered}
$$

for any $T>0$. Moreover if $f_{0}$ belongs to $W^{1, \infty}\left(\mathbb{R}^{2}\right)$, then $f \in W^{1, \infty}\left([0, T] \times \mathbb{R}^{2}\right)$ for any $T>0$.

Actually, in order to obtain more accurate numerical scheme we need to prove more regularity for the above solutions. Employing similar arguments as those in the proof of the previous result it is easily seen that

Theorem 2.2 Assume that $f_{0}$ is non negative, $\left(1+|p|^{k}\right)\left(f_{0}+\left|\nabla_{(x, p)} f_{0}\right|\right)$ belongs to $L^{1}\left(\mathbb{R}^{2}\right)$ (with $k=2$ in the $Q R$ case and $k=1$ in the $F R$ case) and that there is $n_{0}: \mathbb{R} \rightarrow \mathbb{R}_{+}$non decreasing on $\mathbb{R}_{-}$and non increasing on $\mathbb{R}_{+}$such that

$\left(H^{\prime}\right) \quad\left(f_{0}+\left|\nabla_{(x, p)} f_{0}\right|\right)(x, p) \leq n_{0}(p), \quad(x, p) \in \mathbb{R}^{2}$

and $H_{k}, H_{\infty}$ hold true. We suppose also that $\rho_{\mathrm{ext}}$ is non negative, belongs to $W^{1,1}(\mathbb{R}) \cap$ $W^{1, \infty}(\mathbb{R}), E_{0}^{\prime}=\rho_{\text {ext }}-\int_{\mathbb{R}} f_{0} d p, A_{0} \in W^{3, \infty}(\mathbb{R}), A_{1} \in W^{2, \infty}(\mathbb{R})$. Then the unique solution $(f, E, A)$ for the system (1-5) (in the $Q R$ and $F R$ cases) satisfies

$$
\begin{gathered}
f \geq 0, \quad\left(1+|p|^{k}\right)\left(f+\left|\nabla_{(x, p)} f\right|\right) \in L^{\infty}\left([0, T] ; L^{1}\left(\mathbb{R}^{2}\right)\right) \\
\int_{\mathbb{R}}\left(f+\left|\nabla_{(x, p)} f\right|\right)(\cdot, \cdot, p)\left(1+|p|^{k}\right) d p \in L^{\infty}([0, T] \times \mathbb{R}) \\
E \in W^{2, \infty}([0, T] \times \mathbb{R}), \quad A \in W^{3, \infty}([0, T] \times \mathbb{R})
\end{gathered}
$$

for any $T>0$. Moreover if $f_{0}$ belongs to $W^{2, \infty}\left(\mathbb{R}^{2}\right)$, then $f \in W^{2, \infty}\left([0, T] \times \mathbb{R}^{2}\right)$ for any $T>0$.

Corollary 2.1 Under the same hypotheses as in Theorem 2.2, with $H^{\prime}$ replaced by

$$
\left(H^{\prime \prime}\right) \quad\left(f_{0}+\left|\nabla_{(x, p)} f_{0}\right|+\left|\nabla_{(x, p)}^{2} f_{0}\right|\right)(x, p) \leq n_{0}(p), \quad(x, p) \in \mathbb{R}^{2}
$$

we have in addition

$$
\left(1+|p|^{k}\right)\left|\nabla_{(x, p)}^{2} f\right| \in L^{\infty}\left([0, T] ; L^{1}\left(\mathbb{R}^{2}\right)\right), \int_{\mathbb{R}}\left|\nabla_{(x, p)}^{2} f\right|(\cdot, \cdot, p)\left(1+|p|^{k}\right) d p \in L^{\infty}([0, T] \times \mathbb{R})
$$

for any $T>0$ (with $k=2$ in the $Q R$ case and $k=1$ in the FR case). Moreover, if $f_{0}$ has compact support, then $f(t)$ has compact support uniformly with respect to $t \in[0, T]$ for any $T>0$. 


\section{The semi-Lagrangian method for the reduced Vlasov-Maxwell equation}

The semi-Lagrangian method consists in approximating the solution of the Vlasov equation (1) on a phase space grid by using the conservation of the particle distribution along the characteristics. Therefore we need to approximate the characteristics. We use a splitting type method which consists in advecting successively in space and momentum. More precisely the backward characteristic $(X, P)(t-\Delta t ; t, x, p)$ will be approximated by

$$
X(t-\Delta t ; t, x, p) \approx x-\frac{\Delta t}{2} v(p)-\frac{\Delta t}{2} v\left(p-\Delta t F\left(t-\frac{\Delta t}{2}, x-\frac{\Delta t}{2} v(p)\right)\right)
$$

and

$$
P(t-\Delta t ; t, x, p) \approx p-\Delta t F\left(t-\frac{\Delta t}{2}, x-\frac{\Delta t}{2} v(p)\right) .
$$

The expressions for the velocity function and the force field in the QR case are given by

$$
v(p)=\frac{p}{\gamma}=\frac{p}{\left(1+p^{2}\right)^{1 / 2}}, \quad F(t, x)=-\left(E(t, x)+A(t, x) \partial_{x} A(t, x)\right) .
$$

As usual, these formula are obtained by combining three advections. First we perform a one half time step advection along the space variable, secondly we consider a complete time step advection with respect to the momentum variable and we end with another half time step advection along the space variable

$$
\begin{aligned}
(x, p) & \rightarrow\left(x-\frac{\Delta t}{2} v(p), p\right) \\
& \rightarrow\left(x-\frac{\Delta t}{2} v\left(p-\Delta t F\left(t-\frac{\Delta t}{2}, x\right)\right), p-\Delta t F\left(t-\frac{\Delta t}{2}, x\right)\right) \\
& \rightarrow\left(x-\frac{\Delta t}{2} v(p)-\frac{\Delta t}{2} v(p-\Delta t \tilde{F}), p-\Delta t \tilde{F}\right) \\
& =: D_{F(t-\Delta t / 2)}(x, p)
\end{aligned}
$$

where $\tilde{F}=F\left(t-\frac{\Delta t}{2}, x-\frac{\Delta t}{2} v(p)\right)$. In the sequel we denote by $C$ various constants which can change from line to line. By direct computations we check easily the following error estimate.

Lemma 3.1 Assume that $F$ belongs to $W^{2, \infty}([0, T] \times \mathbb{R})$ and let us denote by $(X, P)(s ; t, x, p)$ the characteristics associated to $F$. Consider another force field $F_{h}$ and let $(\tilde{x}, \tilde{p})$ be the backward characteristic given by the splitting method

$$
(\tilde{x}, \tilde{p})=D_{F_{h}(t-\Delta t / 2)}(x, p) .
$$

Then we have

$$
|\tilde{x}-X(t-\Delta t ; t, x, p)| \leq C \Delta t^{3}+C \Delta t^{2}\left\|\left(F-F_{h}\right)(t-\Delta t / 2)\right\|_{L^{\infty}}
$$




$$
|\tilde{p}-P(t-\Delta t ; t, x, p)| \leq C \Delta t^{3}+\Delta t\left\|\left(F-F_{h}\right)(t-\Delta t / 2)\right\|_{L^{\infty}}
$$

Proof. We can write

$$
\begin{aligned}
\tilde{p}-P(t-\Delta t ; t, x, p) & =p-\Delta t F_{h}(t-\Delta t / 2, x-\Delta t v(p) / 2)-p+\int_{t-\Delta t}^{t} F(s, X(s)) \mathrm{d} s \\
& =\int_{t-\Delta t}^{t}\{F(s, X(s))-F(t-\Delta t / 2, X(t-\Delta t / 2))\} \mathrm{d} s \\
& +\Delta t\left(F(t-\Delta t / 2, X(t-\Delta t / 2))-F_{h}(t-\Delta t / 2, x-\Delta t v(p) / 2)\right) .
\end{aligned}
$$

Since $F \in W^{2, \infty}([0, T] \times \mathbb{R})$ we obtain by Taylor expansion of the function $s \rightarrow$ $F(s, X(s))$ around $t-\Delta t / 2$ that

$$
\left|\int_{t-\Delta t}^{t}\{F(s, X(s))-F(t-\Delta t / 2, X(t-\Delta t / 2))\} \mathrm{d} s\right| \leq C \Delta t^{3} .
$$

Observe also that

$$
\begin{aligned}
|x-\Delta t v(p) / 2-X(t-\Delta t / 2)| & =\left|x-\Delta t v(p) / 2-\left(x-\int_{t-\Delta t / 2}^{t} v(P(s)) \mathrm{d} s\right)\right| \\
& =\left|\int_{t-\Delta t / 2}^{t}(v(P(s))-v(p)) \mathrm{d} s\right| \leq C \Delta t^{2}
\end{aligned}
$$

implying that

$$
\begin{aligned}
\mid F(t-\Delta t / 2, X(t-\Delta t / 2)) & -F_{h}(t-\Delta t / 2, x-\Delta t v(p) / 2) \mid \leq\left\|\left(F-F_{h}\right)(t-\Delta t / 2)\right\|_{L^{\infty}} \\
& +|F(t-\Delta t / 2, X(t-\Delta t / 2))-F(t-\Delta t / 2, x-\Delta t v(p) / 2)| \\
& \leq C \Delta t^{2}+\left\|\left(F-F_{h}\right)(t-\Delta t / 2)\right\|_{L^{\infty}} .
\end{aligned}
$$

Combining the previous computations yields

$$
|\tilde{p}-P(t-\Delta t ; t, x, p)| \leq C \Delta t^{3}+\Delta t\left\|\left(F-F_{h}\right)(t-\Delta t / 2)\right\|_{L^{\infty}} .
$$

It remains to compare $\tilde{x}$ and $X(t-\Delta t ; t, x, p)$. We have

$$
\begin{aligned}
\tilde{x}-X(t-\Delta t ; t, x, p) & =x-\frac{\Delta t}{2} v(p)-\frac{\Delta t}{2} v(\tilde{p})-\left(x-\int_{t-\Delta t}^{t} v(P(s)) \mathrm{d} s\right) \\
& =\Delta t\left(v(P(t-\Delta t / 2))-\frac{1}{2} v(p)-\frac{1}{2} v(\tilde{p})\right) \\
& +\int_{t-\Delta t}^{t}(v(P(s))-v(P(t-\Delta t / 2))) \mathrm{d} s .
\end{aligned}
$$

Thanks to the Taylor expansion of the function $s \rightarrow v(P(s))$ around $s=t-\Delta t / 2$ we check easily that

$$
\left|\int_{t-\Delta t}^{t}(v(P(s))-v(P(t-\Delta t / 2))) \mathrm{d} s\right| \leq C \Delta t^{3} .
$$


Notice that we can write

$$
\begin{aligned}
P(t-\Delta t / 2) & =p-\int_{t-\Delta t / 2}^{t} F(s, X(s)) \mathrm{d} s \\
& =p-\frac{\Delta t}{2} F(t-\Delta t / 2, X(t-\Delta t / 2)) \\
& +\int_{t-\Delta t / 2}^{t}(F(t-\Delta t / 2, X(t-\Delta t / 2))-F(s, X(s))) \mathrm{d} s .
\end{aligned}
$$

But we know that

$$
\left|X(t-\Delta t / 2)-\left(x-\frac{\Delta t}{2} v(p)\right)\right| \leq C \Delta t^{2}
$$

and

implying that

$$
\left|\int_{t-\Delta t / 2}^{t}(F(t-\Delta t / 2, X(t-\Delta t / 2))-F(s, X(s))) \mathrm{d} s\right| \leq C \Delta t^{2}
$$

$$
\begin{aligned}
\mid P(t-\Delta t / 2) & -\frac{p+\tilde{p}}{2}|=| P(t-\Delta t / 2)-\left(p-\frac{\Delta t}{2} F_{h}(t-\Delta t / 2, x-\Delta t v(p) / 2)\right) \mid \\
& \leq \frac{\Delta t}{2}\left|F_{h}(t-\Delta t / 2, x-\Delta t v(p) / 2)-F(t-\Delta t / 2, X(t-\Delta t / 2))\right| \\
& +\left|\int_{t-\Delta t / 2}^{t}(F(t-\Delta t / 2, X(t-\Delta t / 2))-F(s, X(s))) \mathrm{d} s\right| \\
& \leq \frac{\Delta t}{2}\left\|\left(F-F_{h}\right)(t-\Delta t / 2)\right\|_{L^{\infty}}+C \Delta t^{2}
\end{aligned}
$$

Combining (7), (8), (9) yields

$$
\begin{aligned}
|\tilde{x}-X(t-\Delta t ; t, x, p)| & \leq \Delta t\left|v((p+\tilde{p}) / 2)-\frac{1}{2} v(p)-\frac{1}{2} v(\tilde{p})\right|+C \Delta t^{3} \\
& +C \Delta t^{2}\left\|\left(F-F_{h}\right)(t-\Delta t / 2)\right\|_{L^{\infty}} .
\end{aligned}
$$

Consider now the function $\varphi(h)=v(p+h / 2)-\frac{1}{2} v(p)-\frac{1}{2} v(p+h)$. By using the Taylor expansion around $h=0$ one gets

$$
\varphi(h)=\frac{h^{2}}{2}\left(\frac{1}{4} v^{\prime \prime}(p+\theta h / 2)-\frac{1}{2} v^{\prime \prime}(p+\theta h)\right), \quad \theta \in(0,1) .
$$

Applying now the above equality with $h=\tilde{p}-p=-\Delta t F_{h}(t-\Delta t / 2, x-\Delta t v(p) / 2)$ we deduce that

$$
\left|v((p+\tilde{p}) / 2)-\frac{1}{2} v(p)-\frac{1}{2} v(\tilde{p})\right| \leq C \Delta t^{2}
$$

and therefore

$$
|\tilde{x}-X(t-\Delta t ; t, x, p)| \leq C \Delta t^{3}+C \Delta t^{2}\left\|\left(F-F_{h}\right)(t-\Delta t / 2)\right\|_{L^{\infty}} .
$$


We introduce now our semi-Lagrangian scheme. We consider the mesh $\left\{\left(x_{i}, p_{j}\right)\right.$ : $\left.(i, j) \in \mathbb{Z}^{2}\right\}$ where $x_{i}=i \Delta x, p_{j}=j \Delta p$ and we denote by

$$
I: l^{\infty}\left(\mathbb{Z}^{2}\right) \rightarrow L^{\infty}\left(\mathbb{R}^{2}\right), \quad \Pi: L^{\infty}\left(\mathbb{R}^{2}\right) \rightarrow l^{\infty}\left(\mathbb{Z}^{2}\right)
$$

a linear interpolation and projection operator respectively. We assume that the interpolation and projection operators satisfy

$$
\|I\|_{\mathcal{L}\left(l^{\infty}\left(\mathbb{Z}^{2}\right), L^{\infty}\left(\mathbb{R}^{2}\right)\right)}=1, \quad \Pi \circ I=\operatorname{Id}_{l \infty\left(\mathbb{Z}^{2}\right)} .
$$

The above operators will be used to approximate the particle distribution function on the mesh. Initially we take $f_{i j}^{0}=f_{0}\left(x_{i}, p_{j}\right)$ for any $(i, j) \in \mathbb{Z}^{2}$ where $f_{0}=f_{0}(x, p)$ is the initial particle distribution. In order to approximate the force field we consider also interpolation and projection operators along the space variable

$$
I_{1}: l^{\infty}(\mathbb{Z}) \rightarrow L^{\infty}(\mathbb{R}), \quad \Pi_{1}: L^{\infty}(\mathbb{R}) \rightarrow l^{\infty}(\mathbb{Z})
$$

satisfying

$$
\left\|I_{1}\right\|_{\mathcal{L}\left(l^{\infty}(\mathbb{Z}), L^{\infty}(\mathbb{R})\right)}=1, \quad \Pi_{1} \circ I_{1}=\operatorname{Id}_{l^{\infty}(\mathbb{Z})} .
$$

We will use the projections

$$
(\Pi u)_{i j}=u\left(x_{i}, p_{j}\right), \quad u \in L^{\infty}\left(\mathbb{R}^{2}\right), \quad(i, j) \in \mathbb{Z}^{2}
$$

and

$$
\left(\Pi_{1} w\right)_{i}=w\left(x_{i}\right), \quad w \in L^{\infty}(\mathbb{R}), \quad i \in \mathbb{Z} .
$$

The operator $I_{1}$ is given by linear interpolation. In this case we have for any function $w \in W^{2, \infty}(\mathbb{R})$

$$
\left\|\left(I_{1} \circ \Pi_{1}\right) w-w\right\|_{L^{\infty}(\mathbb{R})} \leq \frac{\Delta x^{2}}{8}\left\|w^{\prime \prime}\right\|_{L^{\infty}(\mathbb{R})} .
$$

We take as definition for the operator $I$ the following formula: for any $u \in l^{\infty}\left(\mathbb{Z}^{2}\right)$ and $(x, p) \in\left[x_{i}, x_{i+1}\left[\times\left[p_{j}, p_{j+1}[\right.\right.\right.$

$$
\begin{aligned}
(I u)(x, y) & =u_{i, j} \frac{\left(x_{i+1}-x\right)\left(p_{j+1}-p\right)}{\Delta x \Delta p}+u_{i+1, j+1} \frac{\left(x-x_{i}\right)\left(p-p_{j}\right)}{\Delta x \Delta p} \\
& +u_{i, j+1} \frac{\left(x_{i+1}-x\right)\left(p-p_{j}\right)}{\Delta x \Delta p}+u_{i+1, j} \frac{\left(x-x_{i}\right)\left(p_{j+1}-p\right)}{\Delta x \Delta p} .
\end{aligned}
$$

Applying twice the inequality (10) one gets for any function $u \in W^{2, \infty}\left(\mathbb{R}^{2}\right)$

$$
\|(I \circ \Pi) u-u\|_{L^{\infty}\left(\mathbb{R}^{2}\right)} \leq \frac{1}{8}\left(\Delta x^{2}+\Delta p^{2}\right) \max \left\{\left\|\frac{\partial^{2} u}{\partial x^{2}}\right\|_{L^{\infty}},\left\|\frac{\partial^{2} u}{\partial p^{2}}\right\|_{L^{\infty}}\right\} .
$$

At the macroscopic level we take as unknowns the electric field $E$, the potential $A$ but also the derivatives of it, $U=\partial_{t} A, V=\partial_{x} A$. At the continuous level these quantities are related by the equations

$$
\partial_{t} E=\int_{\mathbb{R}} v(p) f d p, \quad \partial_{t} A=U, \partial_{t} U-\partial_{x} V=-A \int_{\mathbb{R}} f d p, \partial_{t} V-\partial_{x} U=0 .
$$


Observe that the last two equalities are equivalent to

$$
\partial_{t}(U+V)-\partial_{x}(U+V)=-A \int_{\mathbb{R}} f d p
$$

and

$$
\partial_{t}(U-V)+\partial_{x}(U-V)=-A \int_{\mathbb{R}} f d p
$$

implying that

$$
\begin{aligned}
U(t, x) & =\frac{1}{2}(U+V)(0, x+t)+\frac{1}{2}(U-V)(0, x-t) \\
& -\frac{1}{2} \int_{0}^{t}(A \rho)(s, x+(t-s)) \mathrm{d} s-\frac{1}{2} \int_{0}^{t}(A \rho)(s, x-(t-s)) \mathrm{d} s
\end{aligned}
$$

and

$$
\begin{aligned}
V(t, x) & =\frac{1}{2}(U+V)(0, x+t)-\frac{1}{2}(U-V)(0, x-t) \\
& -\frac{1}{2} \int_{0}^{t}(A \rho)(s, x+(t-s)) \mathrm{d} s+\frac{1}{2} \int_{0}^{t}(A \rho)(s, x-(t-s)) \mathrm{d} s .
\end{aligned}
$$

We will approximate the potential $A$ on $\left(t^{n}, x_{i}\right)$ and the other fields $E, U, V$ on $\left(t^{n+1 / 2}, x_{i}\right)$. Therefore we initialize the macroscopic fields by

$$
\begin{gathered}
A_{i}^{0}=A_{0}\left(x_{i}\right), \quad E_{i}^{1 / 2}=E_{0}\left(x_{i}\right)+\frac{\Delta t}{2} \sum_{j \in \mathbb{Z}} v\left(p_{j}\right) f_{i j}^{0} \Delta p \\
U_{i}^{1 / 2}=\frac{1}{2}\left(A_{1}\left(x_{i}+\Delta t / 2\right)+A_{0}^{\prime}\left(x_{i}+\Delta t / 2\right)\right)+\frac{1}{2}\left(A_{1}\left(x_{i}-\Delta t / 2\right)-A_{0}^{\prime}\left(x_{i}-\Delta t / 2\right)\right) \\
-\frac{\Delta t}{2} A_{0}\left(x_{i}\right) \sum_{j \in \mathbb{Z}} f_{i j}^{0} \Delta p \\
V_{i}^{1 / 2}=\frac{1}{2}\left(A_{1}\left(x_{i}+\Delta t / 2\right)+A_{0}^{\prime}\left(x_{i}+\Delta t / 2\right)\right)-\frac{1}{2}\left(A_{1}\left(x_{i}-\Delta t / 2\right)-A_{0}^{\prime}\left(x_{i}-\Delta t / 2\right)\right)
\end{gathered}
$$

where $E_{0}, A_{0}, A_{1}$ are the initial conditions for the fields $E, A, \partial_{t} A$. The numerical scheme is given as follows; let us suppose that $f_{i j}^{n}, A_{i}^{n}, E_{i}^{n+1 / 2}, U_{i}^{n+1 / 2}, V_{i}^{n+1 / 2}$ are given for any $(i, j) \in \mathbb{Z}^{2}$ and for some $n \in \mathbb{N}$. Then

- the particle density at the time $t^{n+1}$ is computed by

$$
f_{i j}^{n+1}=\left(I f^{n}\right)\left(D_{F_{h}^{n+1 / 2}}\left(x_{i}, p_{j}\right)\right), \quad(i, j) \in \mathbb{Z}^{2}
$$

where

$$
F_{h}^{n+1 / 2}=-\left(I_{1} E^{n+1 / 2}+I_{1}\left(A^{n}+\frac{\Delta t}{2} U^{n+1 / 2}\right) I_{1} V^{n+1 / 2}\right)
$$


- the potential at the time $t^{n+1}$ is computed by

$$
A_{i}^{n+1}=A_{i}^{n}+\Delta t U_{i}^{n+1 / 2}
$$

- the electric field at the time $t^{n+3 / 2}$ is given by

$$
E_{i}^{n+3 / 2}=E_{i}^{n+1 / 2}+\Delta t \sum_{j \in \mathbb{Z}} v\left(p_{j}\right) f_{i j}^{n+1} \Delta p
$$

- the derivatives of the potential at the time $t^{n+3 / 2}$ are computed by

$(U \pm V)_{i}^{n+3 / 2}=I_{1}\left((U \pm V)^{n+1 / 2}\right)\left(x_{i} \pm \Delta t\right)-\Delta t\left(I_{1}\left(A^{n+1}\right) I_{1}\left(\rho^{n+1}\right)\right)\left(x_{i} \pm \frac{\Delta t}{2}\right), \quad i \in \mathbb{Z}$

where $\rho_{i}^{n+1}=\sum_{j \in \mathbb{Z}} f_{i j}^{n+1} \Delta p$.

For the practical implementation of this scheme we will assume that the initial conditions are compactly supported. By similar arguments as those employed when studying the continuous problem, it is possible to show that if $\left(f_{i j}^{0}\right)_{(i, j) \in \mathbb{Z}^{2}}$ has compact support in $\mathbb{Z}^{2}$, then $\left(f_{i j}^{n}\right)_{(i, j) \in \mathbb{Z}^{2}}$ remains compactly supported in $\mathbb{Z}^{2}$ uniformly with respect to $n \in \mathbb{N}$ such that $n \Delta t \leq T, T \in \mathbb{R}_{+}$.

\section{Convergence study}

In this section we analyze the convergence rate of the numerical scheme previously introduced. We need to estimate the errors

$$
\begin{aligned}
\Delta f_{i j}^{n} & =f_{i j}^{n}-f\left(t^{n}, x_{i}, p_{j}\right) \\
\Delta E_{i}^{n+1 / 2} & =E_{i}^{n+1 / 2}-E\left(t^{n+1 / 2}, x_{i}\right) \\
\Delta A_{i}^{n} & =A_{i}^{n}-A\left(t^{n}, x_{i}\right) \\
\Delta U_{i}^{n+1 / 2} & =U_{i}^{n+1 / 2}-\partial_{t} A\left(t^{n+1 / 2}, x_{i}\right) \\
\Delta V_{i}^{n+1 / 2} & =V_{i}^{n+1 / 2}-\partial_{x} A\left(t^{n+1 / 2}, x_{i}\right)
\end{aligned}
$$

where $(f, E, A)$ is the exact solution of (1-5) (see Theorems 2.1, 2.2). To that purpose we decompose our calculations in several lemmas.

Lemma 4.1 Under the hypotheses of Theorems 2.1, 2.2 there is a constant $C$ such that

$$
\begin{aligned}
\left\|\Delta f^{n+1}\right\|_{l \infty} & \leq\left\|\Delta f^{n}\right\|_{l_{\infty}}+C \Delta t\left(\left\|\Delta E^{n+1 / 2}\right\|_{l_{\infty}}+\left\|\Delta A^{n}\right\|_{l^{\infty}}+\left\|\Delta U^{n+1 / 2}\right\|_{l^{\infty}}+\left\|\Delta V^{n+1 / 2}\right\|_{l^{\infty}}\right) \\
& +C\left(\Delta x^{2}+\Delta p^{2}+\Delta t^{3}+\Delta t \Delta x^{2}\right) .
\end{aligned}
$$


Proof. We can write

$$
\begin{aligned}
\Delta f_{i j}^{n+1} & =\left(I f^{n}\right)\left(D_{F_{h}^{n+1 / 2}}\left(x_{i}, p_{j}\right)\right)-f\left(t^{n+1}, x_{i}, p_{j}\right) \\
& =\left(I f^{n}\right)\left(D_{F_{h}^{n+1 / 2}}\left(x_{i}, p_{j}\right)\right)-f\left(t^{n},(X, P)\left(t^{n} ; t^{n+1}, x_{i}, p_{j}\right)\right) \\
& =f\left(t^{n}, D_{F_{h}^{n+1 / 2}}\left(x_{i}, p_{j}\right)\right)-f\left(t^{n},(X, P)\left(t^{n} ; t^{n+1}, x_{i}, p_{j}\right)\right) \\
& +\left(I f^{n}-f\left(t^{n}\right)\right)\left(D_{F_{h}^{n+1 / 2}}\left(x_{i}, p_{j}\right)\right) .
\end{aligned}
$$

By Lemma 3.1 we have for any $\Delta t<1$

$$
\left|D_{F_{h}^{n+1 / 2}}\left(x_{i}, p_{j}\right)-(X, P)\left(t^{n} ; t^{n+1}, x_{i}, p_{j}\right)\right| \leq C \Delta t^{3}+\Delta t\left\|F_{h}^{n+1 / 2}-F\left(t^{n+1 / 2}\right)\right\|_{L^{\infty}}
$$

where $F_{h}^{n+1 / 2}$ is given by (14) and

$$
F\left(t^{n+1 / 2}, x\right)=-\left(E\left(t^{n+1 / 2}, x\right)+A\left(t^{n+1 / 2}, x\right) \partial_{x} A\left(t^{n+1 / 2}, x\right)\right) .
$$

Since $E \in W^{2, \infty}([0, T] \times \mathbb{R})$ observe by $(10)$ that

$$
\begin{aligned}
\left\|I_{1} E^{n+1 / 2}-E\left(t^{n+1 / 2}\right)\right\|_{L^{\infty}} & \leq\left\|I_{1} E^{n+1 / 2}-\left(I_{1} \circ \Pi_{1}\right) E\left(t^{n+1 / 2}\right)\right\|_{L^{\infty}} \\
& +\frac{\Delta x^{2}}{8}\left\|\partial_{x}^{2} E\left(t^{n+1 / 2}\right)\right\|_{L^{\infty}} \\
& \leq\left\|E^{n+1 / 2}-\Pi_{1} E\left(t^{n+1 / 2}\right)\right\|_{l^{\infty}}+C \Delta x^{2}
\end{aligned}
$$

and similarly, since $A \in W^{3, \infty}([0, T] \times \mathbb{R})$, we obtain

$$
\begin{gathered}
\left\|I_{1} A^{n}-A\left(t^{n}\right)\right\|_{L^{\infty}} \leq\left\|A^{n}-\Pi_{1} A\left(t^{n}\right)\right\|_{l^{\infty}}+C \Delta x^{2} \\
\left\|I_{1} U^{n+1 / 2}-\partial_{t} A\left(t^{n+1 / 2}\right)\right\|_{L^{\infty}} \leq\left\|U^{n+1 / 2}-\Pi_{1} \partial_{t} A\left(t^{n+1 / 2}\right)\right\|_{l^{\infty}}+C \Delta x^{2} \\
\left\|I_{1} V^{n+1 / 2}-\partial_{x} A\left(t^{n+1 / 2}\right)\right\|_{L^{\infty}} \leq\left\|V^{n+1 / 2}-\Pi_{1} \partial_{x} A\left(t^{n+1 / 2}\right)\right\|_{l^{\infty}}+C \Delta x^{2} .
\end{gathered}
$$

Obviously we can write

$$
\begin{aligned}
A\left(t^{n+1 / 2}, x\right) & =A\left(t^{n}, x\right)+\int_{t^{n}}^{t^{n+1 / 2}} \partial_{t} A(s, x) \mathrm{d} s \\
& =A\left(t^{n}, x\right)+\frac{\Delta t}{2} \partial_{t} A\left(t^{n+1 / 2}, x\right)+\int_{t^{n}}^{t^{n+1 / 2}}\left(\partial_{t} A(s, x)-\partial_{t} A\left(t^{n+1 / 2}, x\right)\right) \mathrm{d} s
\end{aligned}
$$

and therefore we obtain

$$
\begin{aligned}
\| F_{h}^{n+1 / 2} & -F\left(t^{n+1 / 2}\right)\left\|_{L^{\infty}} \leq\right\| I_{1} E^{n+1 / 2}-E\left(t^{n+1 / 2}\right) \|_{L^{\infty}} \\
& +\left\|I_{1}\left(A^{n}+\frac{\Delta t}{2} U^{n+1 / 2}\right)\left(I_{1} V^{n+1 / 2}-\partial_{x} A\left(t^{n+1 / 2}\right)\right)\right\|_{L^{\infty}} \\
& +\| \partial_{x} A\left(t^{n+1 / 2}\right)\left(I_{1}\left(A^{n}\right)+\frac{\Delta t}{2} I_{1}\left(U^{n+1 / 2}\right)-A\left(t^{n}\right)-\frac{\Delta t}{2} \partial_{t} A\left(t^{n+1 / 2}\right)\right. \\
& \left.-\int_{t^{n}}^{t^{n+1 / 2}}\left(\partial_{t} A(s, \cdot)-\partial_{t} A\left(t^{n+1 / 2}, \cdot\right)\right) \mathrm{d} s\right) \|_{L^{\infty}} \\
& \leq\left\|E^{n+1 / 2}-\Pi_{1} E\left(t^{n+1 / 2}\right)\right\|_{l^{\infty}}+C\left\|A^{n}-\Pi_{1} A\left(t^{n}\right)\right\|_{l^{\infty}} \\
& +C\left\|V^{n+1 / 2}-\Pi_{1} \partial_{x} A\left(t^{n+1 / 2}\right)\right\|_{l^{\infty}}+C \Delta t\left\|U^{n+1 / 2}-\Pi_{1} \partial_{t} A\left(t^{n+1 / 2}\right)\right\|_{l^{\infty}} \\
& +C\left(\Delta t^{2}+\Delta x^{2}\right) .
\end{aligned}
$$


But $f \in W^{2, \infty}\left([0, T] \times \mathbb{R}^{2}\right)$ and thus combining (11), (16), (17), (21) implies

$$
\begin{aligned}
\left|\Delta f_{i j}^{n+1}\right| & \leq\left\|I f^{n}-(I \circ \Pi) f\left(t^{n}\right)\right\|_{L^{\infty}}+\left\|(I \circ \Pi) f\left(t^{n}\right)-f\left(t^{n}\right)\right\|_{L^{\infty}} \\
& +C\left|D_{F_{h}^{n+1 / 2}}\left(x_{i}, p_{j}\right)-(X, P)\left(t^{n} ; t^{n+1}, x_{i}, p_{j}\right)\right| \\
& \leq\left\|f^{n}-\Pi f\left(t^{n}\right)\right\|_{l^{\infty}}+C\left(\Delta x^{2}+\Delta p^{2}\right)+C \Delta t^{3} \\
& +C \Delta t\left\|E^{n+1 / 2}-\Pi_{1} E\left(t^{n+1 / 2}\right)\right\|_{l^{\infty}}+C \Delta t\left\|A^{n}-\Pi_{1} A\left(t^{n}\right)\right\|_{l^{\infty}} \\
& +C \Delta t\left\|V^{n+1 / 2}-\Pi_{1} \partial_{x} A\left(t^{n+1 / 2}\right)\right\|_{l^{\infty}}+C \Delta t^{2}\left\|U^{n+1 / 2}-\Pi_{1} \partial_{t} A\left(t^{n+1 / 2}\right)\right\|_{l^{\infty}} \\
& +C \Delta t\left(\Delta t^{2}+\Delta x^{2}\right)
\end{aligned}
$$

and our conclusion follows.

Lemma 4.2 Under the hypotheses of Theorems 2.1, 2.2, there is a constant $C$ such that

$$
\left\|\Delta A^{n+1}\right\|_{l^{\infty}} \leq\left\|\Delta A^{n}\right\|_{l^{\infty}}+\Delta t\left\|\Delta U^{n+1 / 2}\right\|_{l^{\infty}}+C \Delta t^{3} .
$$

Proof. We have

$$
A\left(t^{n+1}, x_{i}\right)=A\left(t^{n}, x_{i}\right)+\int_{t^{n}}^{t^{n+1}} \partial_{t} A\left(s, x_{i}\right) \mathrm{d} s
$$

and therefore

$$
\begin{aligned}
\Delta A_{i}^{n+1} & =A_{i}^{n}+\Delta t U_{i}^{n+1 / 2}-A\left(t^{n}, x_{i}\right)-\Delta t \partial_{t} A\left(t^{n+1 / 2}, x_{i}\right) \\
& -\int_{t^{n}}^{t^{n+1}}\left(\partial_{t} A\left(s, x_{i}\right)-\partial_{t} A\left(t^{n+1 / 2}, x_{i}\right)\right) \mathrm{d} s .
\end{aligned}
$$

We deduce that

$$
\left|\Delta A_{i}^{n+1}\right| \leq\left|\Delta A_{i}^{n}\right|+\Delta t\left|\Delta U_{i}^{n+1 / 2}\right|+C \Delta t^{3}
$$

Lemma 4.3 Under the hypotheses of Theorems 2.1, 2.2 and Corollary 2.1, there is a constant $C$ such that

$$
\left\|\Delta E^{n+3 / 2}\right\|_{l^{\infty}} \leq\left\|\Delta E^{n+1 / 2}\right\|_{l^{\infty}}+C \Delta t\left\|\Delta f^{n+1}\right\|_{l^{\infty}}+C \Delta t \Delta p^{2}+C \Delta t^{3} .
$$

Proof. Since the particle density $f$ has compact support and belongs to $W^{2, \infty}([0, T] \times$ $\mathbb{R}^{2}$ ) we have

$$
\begin{aligned}
& \left|\int_{\mathbb{R}} v(p) f\left(t^{n+1}, x_{i}, p\right) d p-\sum_{j \in \mathbb{Z}} v\left(p_{j}\right) f\left(t^{n+1}, x_{i}, p_{j}\right) \Delta p\right| \\
= & \left|\sum_{j \in \mathbb{Z}} \int_{\left|p-p_{j}\right|<\Delta p / 2}\left(v(p) f\left(t^{n+1}, x_{i}, p\right)-v\left(p_{j}\right) f\left(t^{n+1}, x_{i}, p_{j}\right)\right) \mathrm{d} p\right| \\
\leq & \sum_{j \in \mathbb{Z}} \int_{\left|p-p_{j}\right|<\Delta p / 2} \frac{1}{2}\left(p-p_{j}\right)^{2}\left\|\frac{\partial^{2}}{\partial p^{2}}\left\{v(\cdot) f\left(t^{n+1}, x_{i}, \cdot\right)\right\}\right\|_{L^{\infty}} \mathrm{d} p \leq C \Delta p^{2} .
\end{aligned}
$$


Therefore we obtain

$$
\begin{aligned}
\left|\Delta E_{i}^{n+3 / 2}\right| & =\left|\Delta E_{i}^{n+1 / 2}+\Delta t \sum_{j \in \mathbb{Z}} v\left(p_{j}\right) f_{i j}^{n+1} \Delta p-\int_{t^{n+1 / 2}}^{t^{n+3 / 2}} \int_{\mathbb{R}} v(p) f\left(s, x_{i}, p\right) d p \mathrm{~d} s\right| \\
& \leq\left|\Delta E_{i}^{n+1 / 2}\right|+\Delta t\left|\sum_{j \in \mathbb{Z}} v\left(p_{j}\right)\left(f_{i j}^{n+1}-f\left(t^{n+1}, x_{i}, p_{j}\right)\right) \Delta p\right| \\
& +\Delta t\left|\sum_{j \in \mathbb{Z}} v\left(p_{j}\right) f\left(t^{n+1}, x_{i}, p_{j}\right) \Delta p-\int_{\mathbb{R}} v(p) f\left(t^{n+1}, x_{i}, p\right) d p\right| \\
& +\left|\int_{t^{n+1 / 2}}^{t^{n+3 / 2}} \int_{\mathbb{R}} v(p)\left(f\left(s, x_{i}, p\right)-f\left(t^{n+1}, x_{i}, p\right)\right) d p \mathrm{~d} s\right| \\
& \leq\left|\Delta E_{i}^{n+1 / 2}\right|+C \Delta t \Delta p^{2}+C \Delta t^{3}+\Delta t \sum_{j \in \mathbb{Z}}\left|\Delta f_{i j}^{n+1}\right| \Delta p .
\end{aligned}
$$

Our conclusion follows immediately since $f^{n+1}$ and $f\left(t^{n+1}\right)$ have compact support with respect to $(i, j)$ and $(x, p)$ respectively and thus

$$
\sum_{j \in \mathbb{Z}}\left|\Delta f_{i j}^{n+1}\right| \Delta p \leq C\left\|\Delta f^{n+1}\right\|_{l^{\infty}} .
$$

Lemma 4.4 Under the hypotheses of Theorems 2.1, 2.2 and Corollary 2.1 there is a constant $C$ such that

$$
\begin{aligned}
\left\|\Delta\left(U^{n+3 / 2} \pm V^{n+3 / 2}\right)\right\|_{l^{\infty}} & \leq\left\|\Delta\left(U^{n+1 / 2} \pm V^{n+1 / 2}\right)\right\|_{l^{\infty}}+C \Delta t\left(\left\|\Delta f^{n+1}\right\|_{l^{\infty}}+\left\|\Delta A^{n+1}\right\|_{l^{\infty}}\right) \\
& +C \Delta x^{2}+C \Delta t\left(\Delta x^{2}+\Delta p^{2}\right) .
\end{aligned}
$$

Proof. We have

$$
\begin{aligned}
\left(\partial_{t} A \pm \partial_{x} A\right)\left(t^{n+3 / 2}, x_{i}\right) & =\left(\partial_{t} A \pm \partial_{x} A\right)\left(t^{n+1 / 2}, x_{i} \pm \Delta t\right) \\
& -\int_{t^{n+1 / 2}}^{t^{n+3 / 2}} A\left(s, x_{i} \pm\left(t^{n+3 / 2}-s\right)\right) \rho\left(s, x_{i} \pm\left(t^{n+3 / 2}-s\right)\right) \mathrm{d} s \\
& =\left(\partial_{t} A \pm \partial_{x} A\right)\left(t^{n+1 / 2}, x_{i} \pm \Delta t\right)-\Delta t(A \rho)\left(t^{n+1}, x_{i} \pm \Delta t / 2\right) \\
& -\int_{t^{n+1 / 2}}^{t^{n+3 / 2}}\left((A \rho)\left(s, x_{i} \pm\left(t^{n+3 / 2}-s\right)\right)-(A \rho)\left(t^{n+1}, x_{i} \pm \Delta t / 2\right)\right) \mathrm{d} s
\end{aligned}
$$

Therefore we deduce that

$$
\begin{aligned}
\left|\Delta\left(U_{i}^{n+3 / 2} \pm V_{i}^{n+3 / 2}\right)\right| & \leq\left\|I_{1}\left(U^{n+1 / 2} \pm V^{n+1 / 2}\right)-\left(\partial_{t} A\left(t^{n+1 / 2}\right) \pm \partial_{x} A\left(t^{n+1 / 2}\right)\right)\right\|_{L^{\infty}} \\
& +\Delta t\left\|I_{1}\left(A^{n+1}\right) I_{1}\left(\rho^{n+1}\right)-A\left(t^{n+1}\right) \rho\left(t^{n+1}\right)\right\|_{L^{\infty}}+C \Delta t^{3} .
\end{aligned}
$$


As usual we have the estimate

$$
\left\|I_{1}\left(\rho^{n+1}\right)-\rho\left(t^{n+1}\right)\right\|_{L^{\infty}} \leq\left\|\rho^{n+1}-\Pi_{1} \rho\left(t^{n+1}\right)\right\|_{l^{\infty}}+C \Delta x^{2} .
$$

Taking into account that $\partial_{p}^{2} f \in L^{\infty}\left([0, T] \times \mathbb{R}^{2}\right)$, observe that for any $i \in \mathbb{Z}$ we can write

$$
\begin{aligned}
\left|\rho_{i}^{n+1}-\rho\left(t^{n+1}, x_{i}\right)\right| & =\left|\sum_{j \in \mathbb{Z}} f_{i j}^{n+1} \Delta p-\int_{\mathbb{R}} f\left(t^{n+1}, x_{i}, p\right) d p\right| \\
& \leq \sum_{j \in \mathbb{Z}}\left|\int_{\left|p-p_{j}\right|<\Delta p / 2}\left(f\left(t^{n+1}, x_{i}, p\right)-f\left(t^{n+1}, x_{i}, p_{j}\right)\right) \mathrm{d} p\right| \\
& +\sum_{j \in \mathbb{Z}}\left|f\left(t^{n+1}, x_{i}, p_{j}\right)-f_{i j}^{n+1}\right| \Delta p \\
& \leq\left\|\Delta f^{n+1}\right\|_{l \infty}+C \Delta p^{2} .
\end{aligned}
$$

Thus we obtain

$$
\left\|I_{1}\left(\rho^{n+1}\right)-\rho\left(t^{n+1}\right)\right\|_{L^{\infty}} \leq\left\|\Delta f^{n+1}\right\|_{l^{\infty}}+C\left(\Delta x^{2}+\Delta p^{2}\right)
$$

which implies, by combining with (18) (written for $n+1),(19),(20)$ that

$$
\begin{aligned}
\left\|\Delta\left(U^{n+3 / 2} \pm V^{n+3 / 2}\right)\right\|_{l^{\infty}} & \leq\left\|\Delta\left(U^{n+1 / 2} \pm V^{n+1 / 2}\right)\right\|_{l^{\infty}}+C \Delta x^{2} \\
& +C \Delta t\left(\left\|\Delta A^{n+1}\right\|_{l^{\infty}}+C \Delta x^{2}\right) \\
& +C \Delta t\left(\left\|\Delta f^{n+1}\right\|_{l^{\infty}}+C \Delta x^{2}+C \Delta p^{2}\right) .
\end{aligned}
$$

Finally we evaluate the global error $\Delta^{n+1}$ defined by

$$
\begin{aligned}
e^{n} & :=\left\|\Delta f^{n}\right\|_{l^{\infty}}+\left\|\Delta A^{n}\right\|_{l^{\infty}}+\left\|\Delta E^{n+1 / 2}\right\|_{l^{\infty}} \\
& +\left\|\Delta\left(U^{n+1 / 2}+V^{n+1 / 2}\right)\right\|_{l^{\infty}}+\left\|\Delta\left(U^{n+1 / 2}-V^{n+1 / 2}\right)\right\|_{l^{\infty}} .
\end{aligned}
$$

Theorem 4.1 Under the hypotheses of Theorems 2.1, 2.2 and Corollary 2.1 there is a constant $C$ such that for any $n \in\{0,1, \ldots, N\}$ with $T=N \Delta t$ we have

$$
e^{n} \leq C\left(\Delta t^{2}+\Delta x^{2}+\Delta p^{2}+\Delta t \Delta p^{2}\right)+C \frac{\Delta x^{2}+\Delta p^{2}}{\Delta t}
$$

Proof. By adding the partial error estimates obtained in the Lemmas 4.1, 4.2, 4.3, 4.4 we obtain

$$
\begin{aligned}
e^{n+1} \leq & e^{n}+C \Delta t\left(e^{n}+\left\|\Delta f^{n+1}\right\|_{l^{\infty}}+\left\|\Delta A^{n+1}\right\|_{l^{\infty}}\right) \\
& +C\left(\Delta x^{2}+\Delta p^{2}+\Delta t^{3}+\Delta t \Delta x^{2}+\Delta t \Delta p^{2}\right) .
\end{aligned}
$$


Using one more time the Lemmas 4.1, 4.2 in the above inequality one gets

$$
e^{n+1} \leq e^{n}+C \Delta t e^{n}+C\left(\Delta x^{2}+\Delta p^{2}\right)+C \Delta t\left(\Delta t^{2}+\Delta x^{2}+\Delta p^{2}\right)
$$

implying that

$$
\begin{aligned}
e^{n} & \leq \exp (n C \Delta t) e^{0}+\exp (n C \Delta t)\left(\Delta x^{2}+\Delta p^{2}+\Delta t\left(\Delta t^{2}+\Delta x^{2}+\Delta p^{2}\right)\right) / \Delta t \\
& \leq \exp (C T) e^{0}+\exp (C T)\left(\frac{\Delta x^{2}+\Delta p^{2}}{\Delta t}+\Delta t^{2}+\Delta x^{2}+\Delta p^{2}\right) .
\end{aligned}
$$

It remains to observe that $\left\|\Delta f^{0}\right\|_{l^{\infty}}=\left\|\Delta A^{0}\right\|_{l^{\infty}}=0$ and to estimate $\left\|\Delta E^{1 / 2}\right\|_{l^{\infty}}, \| \Delta(U \pm$ $V)^{1 / 2} \|_{l^{\infty}}$. Obviously we have

$$
\begin{aligned}
\Delta E_{i}^{1 / 2} & =E_{0}\left(x_{i}\right)+\frac{\Delta t}{2} \sum_{j \in \mathbb{Z}} v\left(p_{j}\right) f_{i j}^{0} \Delta p-E\left(t^{1 / 2}, x_{i}\right) \\
& =\frac{\Delta t}{2} \sum_{j \in \mathbb{Z}} v\left(p_{j}\right) f_{0}\left(x_{i}, p_{j}\right) \Delta p-\int_{0}^{t^{1 / 2}} \int_{\mathbb{R}} v(p) f\left(s, x_{i}, p\right) d p \mathrm{~d} s \\
& =\frac{\Delta t}{2} \sum_{j \in \mathbb{Z}} v\left(p_{j}\right) f_{0}\left(x_{i}, p_{j}\right) \Delta p-\frac{\Delta t}{2} \int_{\mathbb{R}} v(p) f_{0}\left(x_{i}, p\right) d p \\
& -\int_{0}^{t^{1 / 2}} \int_{\mathbb{R}} v(p)\left(f\left(s, x_{i}, p\right)-f\left(0, x_{i}, p\right)\right) d p \mathrm{~d} s
\end{aligned}
$$

implying that

$$
\begin{aligned}
\left\|\Delta E^{1 / 2}\right\|_{l^{\infty}} & \leq \frac{\Delta t}{2}\left|\sum_{j \in \mathbb{Z}} \int_{\left|p-p_{j}\right|<\Delta p / 2}\left(v\left(p_{j}\right) f_{0}\left(x_{i}, p_{j}\right)-v(p) f_{0}\left(x_{i}, p\right)\right) \mathrm{d} p\right|+C \Delta t^{2} \\
& \leq C \Delta t \Delta p^{2}+C \Delta t^{2} .
\end{aligned}
$$

By the definitions of $U^{1 / 2}, V^{1 / 2}$ we have

$$
\begin{aligned}
\Delta(U \pm V)^{1 / 2} & =-\frac{\Delta t}{2} A_{0}\left(x_{i}\right) \sum_{j \in \mathbb{Z}} f_{0}\left(x_{i}, p_{j}\right) \Delta p+\int_{0}^{t^{1 / 2}}(A \rho)\left(s, x_{i} \pm\left(t^{1 / 2}-s\right)\right) \mathrm{d} s \\
& =-\frac{\Delta t}{2} A_{0}\left(x_{i}\right)\left(\sum_{j \in \mathbb{Z}} f_{0}\left(x_{i}, p_{j}\right) \Delta p-\int_{\mathbb{R}} f_{0}\left(x_{i}, p\right) d p\right) \\
& +\int_{0}^{t^{1 / 2}}\left\{(A \rho)\left(s, x_{i} \pm\left(t^{1 / 2}-s\right)\right)-(A \rho)\left(0, x_{i}\right)\right\} \mathrm{d} s .
\end{aligned}
$$

Observe that for any $s \in\left[0, t^{1 / 2}\right]$ we have

$$
\left|(\rho A)\left(0, x_{i}\right)-(\rho A)\left(s, x_{i} \pm\left(t^{1 / 2}-s\right)\right)\right| \leq C \Delta t
$$


and that

$$
\left|\sum_{j \in \mathbb{Z}} f_{0}\left(x_{i}, p_{j}\right) \Delta p-\int_{\mathbb{R}} f_{0}\left(x_{i}, p\right) d p\right| \leq C \Delta p^{2} .
$$

Therefore we obtain

$$
\left\|\Delta(U \pm V)^{1 / 2}\right\|_{l^{\infty}} \leq C \Delta t^{2}+C \Delta t \Delta p^{2}
$$

implying that

$$
e^{0} \leq C\left(\Delta t^{2}+\Delta t \Delta p^{2}\right)
$$

and finally one gets

$$
e^{n} \leq C\left(\Delta t^{2}+\Delta x^{2}+\Delta p^{2}+\Delta t \Delta p^{2}\right)+C \frac{\Delta x^{2}+\Delta p^{2}}{\Delta t} .
$$

\section{$5 \quad$ Numerical results}

In this section, some numerical results obtained with the numerical scheme presented above are exposed and compared to analytical solutions when there are available.

\subsection{Non relativistic Vlasov-Maxwell model}

The model considered in this subsection is the so-called NR Vlasov-Maxwell model since $\gamma_{1}=\gamma_{2}=1$ in (1)-(4). In the simplified homogeneous case, it is possible to compute solutions. Indeed, the model reduces to

$$
\begin{gathered}
\partial_{t} f-E(t) \partial_{p} f=0 \\
\partial_{t} E=j(t)
\end{gathered}
$$

where $j(t)=\int_{\mathbb{R}} f(t, p) d p$. By integrating (22) to get an equation on $\rho(t)=\int f(t, p) d p$ with respect to $p$, we obtain $\partial_{t} \rho=0$, which leads to $\rho(t)=\rho(0)(=1$ after normalization). Then considering the equation on $j$ from the integration of (22) leads to the following system

$$
\left\{\begin{array}{l}
\partial_{t} j+E=0 \\
\partial_{t} E-j=0
\end{array}\right.
$$

the solution of which writes

$$
j(t)=j(0) \cos t, \quad E(t)=j(0) \sin t .
$$

Then, to simulate the Vlasov-Maxwell model, we consider an homogeneous initial condition for $f$

$$
f(t=0, x, p)=\frac{1}{\sqrt{2 \pi}} \exp \left(-\frac{|v-j(0)|^{2}}{2}\right),
$$


which leads to the following analytical solution

$$
f(t, x, p)=\frac{1}{\sqrt{2 \pi}} \exp \left(-\frac{|v-j(t)|^{2}}{2}\right) .
$$

The numerical results then will be compared to the analytical current and electric field (25).

To that purpose, the following numerical parameters are considered: $x \in[0,1]$, $p \in[-6-|j(0)|, 6+|j(0)|]$ which ensures that the distribution is well described, whereas $N_{x}=128$ and $N_{p}=128$ are the number of points used to discretize the phase space domain. Finally, the time step is equal to $d t=\pi \times 10^{-3}$ and the initial current is $j(0)=1$.

On figure 1 , we plot the numerical macroscopic current $j(t, x)=\int_{\mathbb{R}} f(t, x, p)$ for different times $t=0, \pi / 2, \pi$; we observe that the numerical solution does not depend on the space variable $x$. Then, on figures 2 and 3 , comparisons between numerical and analytical solutions are performed. The current $j$ and the electric field $E$ are plotted for every time steps, and for $x=0.5$. These numerical results are superimposed with the corresponding analytical solution given by (25). We observe that the two curves are in very good agreement with analytical solutions. These observations are confirmed in figure 4 in which the error $e(t)$ defined by

$$
e(t)=\left|\cos t-j_{\text {num }}(t, x=0.5)\right|
$$

is plotted as a function of time, for different number of points per direction. We can observe that the number of points has an important effect on the behavior of the error. Finally, Fig. 5 compares the cubic spline interpolation and the Lagrange interpolation. The numerical scheme remains unchanged except that a different interpolation operator is used. The error generated by the cubic spline operator is very small compared to that of the Lagrange one. It confirms the fact that the Lagrange interpolation is very diffusive whereas cubic spline represents a good compromise between complexity and accuracy (see [19]).

Finally, the last two curves represent the $L^{1}$ norm of the difference between the analytical and the numerical solution.

\subsection{Fully relativistic Vlasov-Maxwell model}

This subsection presents a more realistic test case from a physical of view since this fully relativistic model can be used for the modelling of laser-plasma interaction problems $([14,15,16])$. Since the previous numerical scheme is too diffusive (due to the creation of very thin structures), a more precise interpolation operator has to be employed. Then, following the observations of the previous subsection, the interpolation operator is replaced by a cubic splines one. The proof can be extended to this context using tools developed in [2]. However, the rest of the algorithm remains similar (the computation of the feet of the characteristics is performed as in section 3 for example). 


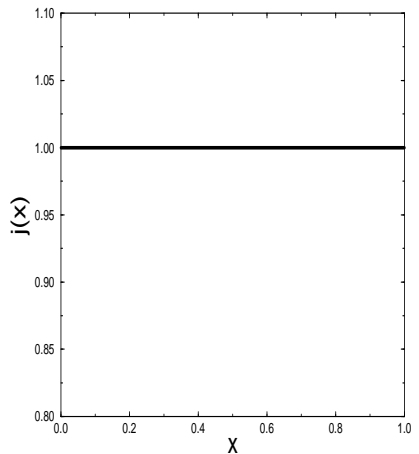

(a)

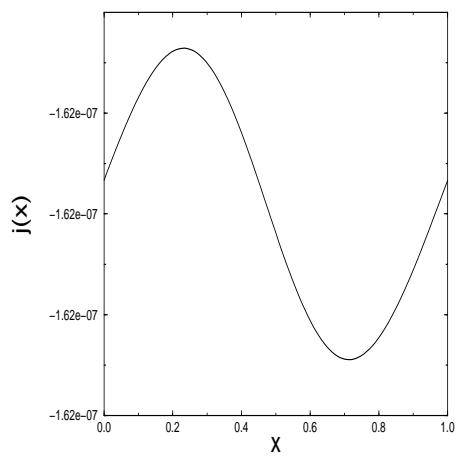

(b)

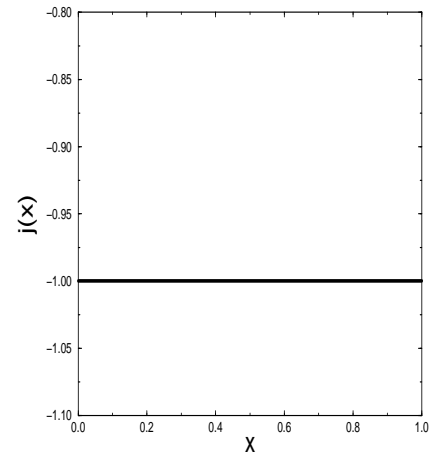

(c)

Figure 1: Macroscopic current as a function of the space variable for $(a) t=$ 0, (b) $t=\pi / 2,(c) t=\pi$.

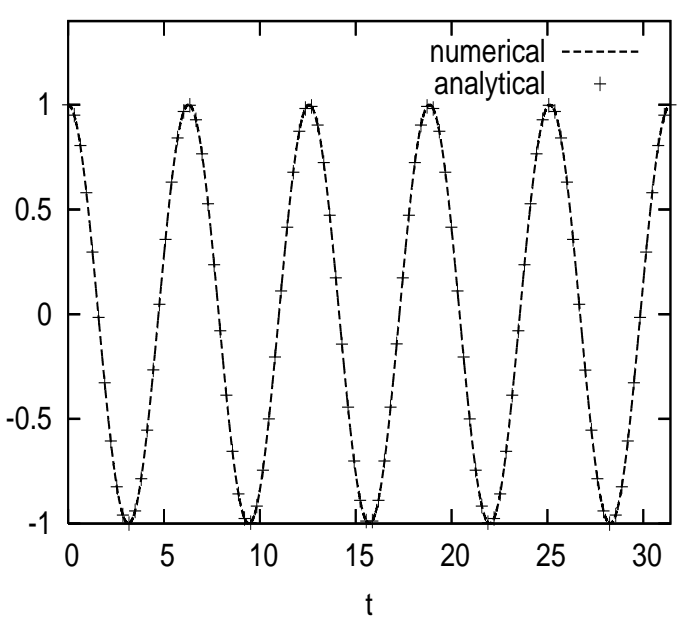

Figure 2: Comparison between the current $j(t)$ and the analytical solution $j(0) \cos t$, as a funcion of time. 


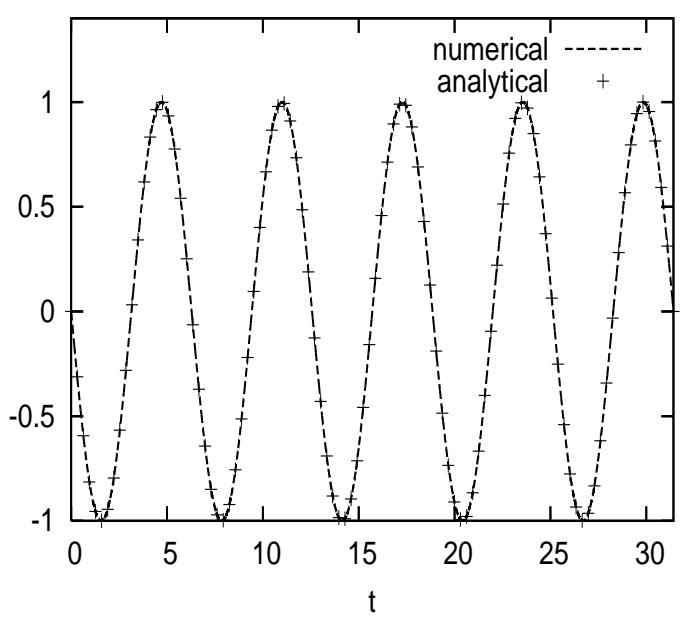

Figure 3: Comparison between the electric field $E(t)$ and the analytical solution $-j(0) \sin t$, as a function of time.

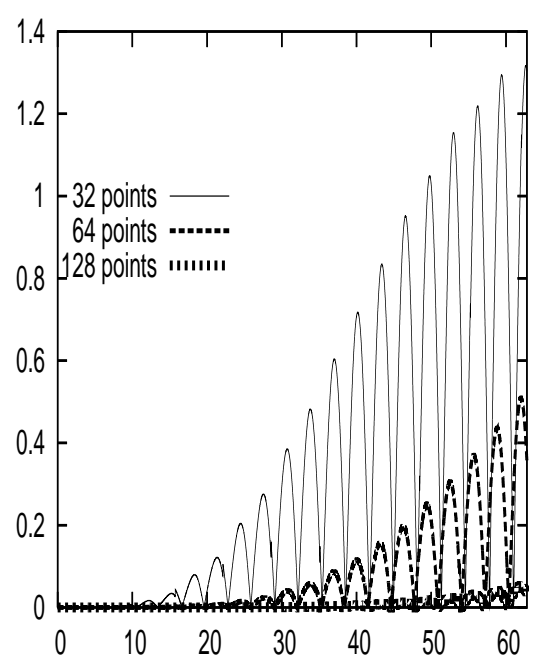

Figure 4: Error between the analytical and the numerical solutions of the current as a function of time, for different number of points per direction. $\Delta t=\pi \times 10^{-3}$ 


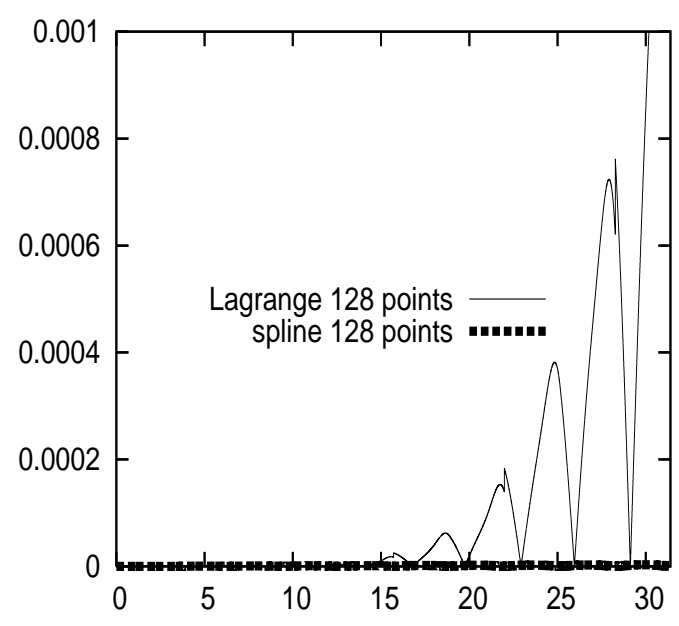

Figure 5: Error between the analytical and the numerical solutions of the current as a function of time: comparison between the cubic spline interpolation and the Lagrange interpolation, with 128 points per direction, $\Delta t=\pi \times 10^{-3}$.

The model here takes into account relativistic effects with

$$
\gamma_{1}=\gamma_{2}=\sqrt{1+p^{2}+A_{y}(t, x)+A_{z}(t, x)} .
$$

The initial condition describes a circularly polarized electromagnetic field:

$$
\begin{array}{ll}
U_{y}^{0}(x)=E_{0} \cos \left(k_{0} x\right) & U_{z}^{0}(x)=E_{0} \sin \left(k_{0} x\right) \\
V_{y}^{0}(x)=-\frac{k_{0} E_{0}}{\omega_{0}} \sin \left(k_{0} x\right) & V_{z}^{0}(x)=\frac{k_{0}^{\star} E_{0}}{\omega_{0}} \cos \left(k_{0} x\right) \\
A_{y}^{0}(x)=-\frac{E_{0}}{\omega_{0}} \sin \left(k_{0} x\right) & A_{z}^{0}(x)=\frac{E_{0}}{\omega_{0}} \cos \left(k_{0} x\right) \\
E^{-1 / 2}=-\frac{\Delta t}{2} \sum_{j \in \mathbb{Z}} v\left(p_{j}\right) f_{i j}^{n+1} \Delta p_{j} . &
\end{array}
$$

The physical parameters are chosen as follows:

$$
\gamma_{0}=2, \omega_{0}=1, k_{0}=1 / \sqrt{2}, E_{0}=\sqrt{3} \omega_{0} .
$$

Finally, the initial distribution function is an homogeneous Maxwellian with a temperature $T=3 / 511$

$$
f_{0}(x, p)=\frac{1}{\sqrt{2 \pi T}} \exp \left(-p^{2} / T\right)
$$

The space domain is taken equal to $\left[0,2 \pi / k_{0}\right]$ whereas the impulsion domain is equal to $\left[-p_{\max }, p_{\max }\right]$, with $p_{\max }=2.5$. The number of grid points is equal to 128 in the physical space whereas 256 points are considered in the impulsion domain. Finally, the time step is $d t=0.01$ and the simulation runs during 10000 time steps. 


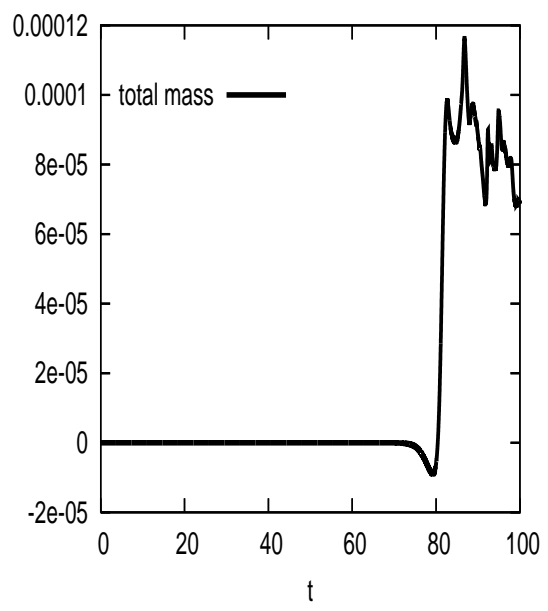

Figure 6: Time evolution of the total mass.

To summarize, the algorithm described at the end of section 3 is applied to the relativistic Vlasov-Maxwell model. The initial conditions represent an unstable stationary solution that will be perturbed by round off errors; hence the growth rate of the numerical solution can be compared to the analytical growth rates which is computed by solving the dispersion relation (see $[14,10]$ ).

In figures 6 and 7, conserved quantities (total mass and total energy) are plotted as a function of time. We can observed that the total mass is preserved with a precision of $0.1 \%$; on the other side, the total mass presents strong oscillations when the instability occurs. In figures 8 and 9 , the time evolution of the most unstable plasma mode (mode 2) is plotted on a logarithmic scale. The linear growth rate can be compared to the imaginary part of the dispersion relation solution. The numerical growth rate is about 0.35 for $U_{y}$ and 0.4 for $E$ which is in quite good agreement with the theoritical growth rate $g \approx 0.409$.

Finally, Fig. 10 represents the electron distribution fonction in phase space. We see the instability begins at time $t \approx 80 \omega_{p}^{-1}$ whereas nothing happens before this time. On the contrary, after the instability begins, very thin structures appears and moves rapidly in the phase space. Cubic spline interpolation appears to be relevant.

\section{Conclusion}

In this work, we studied the convergence of a semi-Lagrangian numerical scheme dedicated to the approximation of one dimensional Vlasov-Maxwell systems. Using an order two numerical scheme to solve the characteristics backward together with a linear Lagrange interpolation operator enable to prove that the numerical scheme converges towards the solution of the model, the existence and unicity of which has been proven in [6]. However, semi-Lagrangian methods require high order interpolation operators in practice (see [13]) to get accurate numerical solutions. Even if 


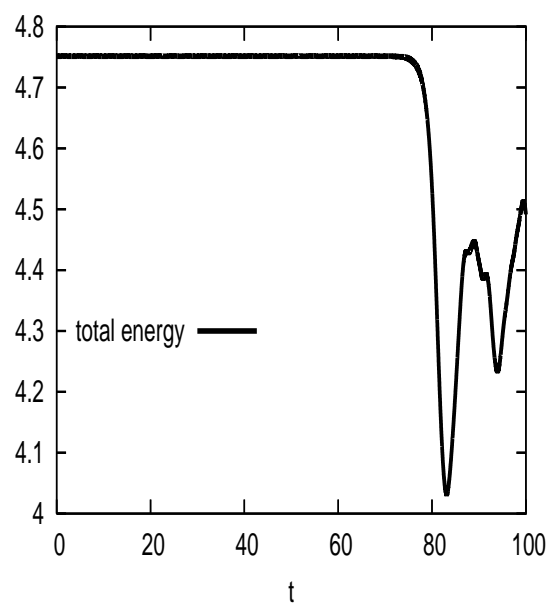

Figure 7: Time evolution of the total energy.

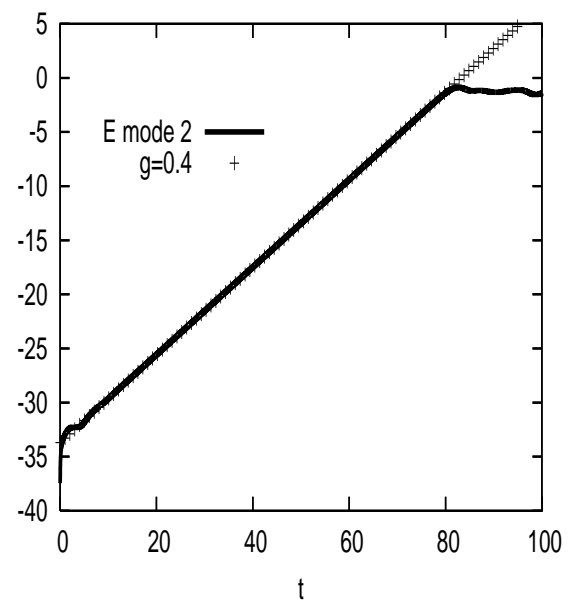

Figure 8: Time evolution of the second mode of E.

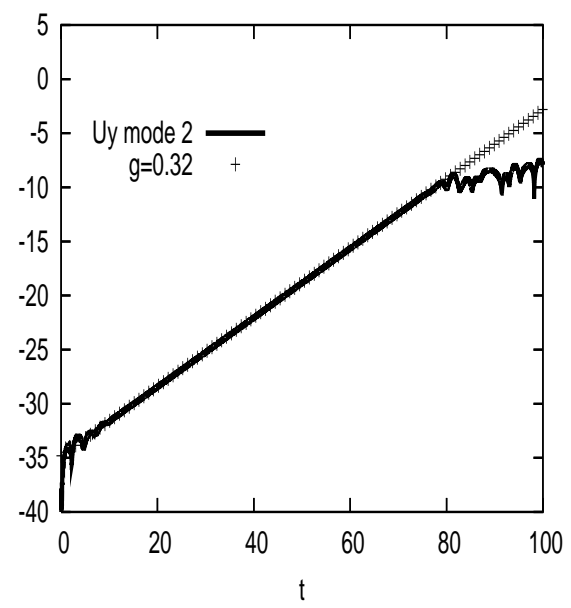

Figure 9: Time evolution of the second mode of $U_{y}$. 

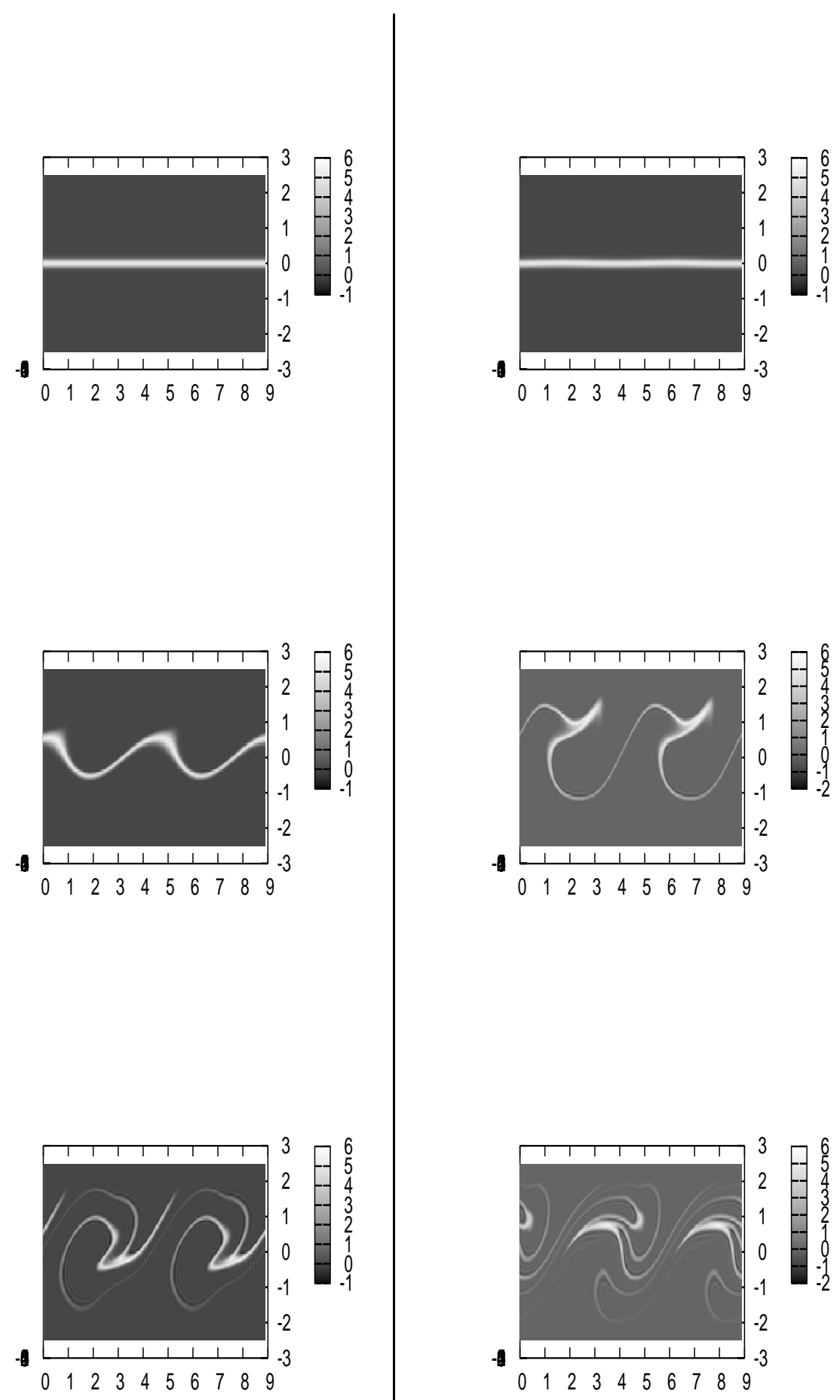

Figure 10: Distribution function in the phase space at diferent times; from top-left to down-right: $t=0,70,80,84,88,92 \omega_{p}^{-1}$. 
linear Lagrange operator seems to be sufficient in some cases, for more realistic numerical experiments however, higher order operators (like cubic spline interpolation operator for example) have been used to capture thin structures or to control the diffusivity. This has been performed in the laser-plasma interaction context and prove the efficiency of the algorithm.

\section{References}

[1] N. Besse, Convergence of a semi-Lagrangian scheme for the Vlasov-Poisson system, SIAM J. Numer. Anal. 42(2004), pp. 350-382.

[2] N. Besse, M. Mehrenberger, Convergence of a classes of high order semiLagrangian schemes for the Vlasov equation, to appear in Math. Comput.

[3] N. Besse, E. SonnendrüCKer, Semi-Lagrangian schemes for the Vlasov equation on an unstructured mesh of phase space, J. Comput. Phys. 191(2003), pp. 341-376.

[4] C.K. Birdsall, A.B. Langdon, Plasma Physics via Computer Simulation, Institute of Physics Publishing, Bristol and Philadelphia, 1991.

[5] J.-P. Boris, D.L. Book, Solution of continuity equations by the method of flux-corrected transport, J. Comput. Phys. 20(1976), pp. 397-431.

[6] M. Bostan, Mild solutions for the relativistic Vlasov-Maxwell system for laser-plasma interaction, Quart. Appl. Math. LXV(2007), pp. 163-187.

[7] M. Campos-Pinto, M. Mehrenberger, Convergence of an adaptive scheme for the one-dimensional Vlasov-Poisson system, Rapport de Recherche INRIA 5519(2005).

[8] Carillo, S. Labrunie, Global solutions for the one-dimensional VlasovMaxwell system for laser-plasma interaction, Math. Models Methods Appl. Sci. 16(2006), pp. 19-57.

[9] C.Z. Cheng, G. Knorr, The integration of the Vlasov equation in configuration space, J. Comput. Phys. 22(1976), pp. 330-351.

[10] N. Crouseilles, A. Ghizzo, S. Salmon, Vlasov laser-plasma interaction simulations in the relativistic regime with a moving grid, Research Report Number 6109, 2007.

[11] F. Filbet, Convergence of a Finite Volume Scheme for the One Dimensional Vlasov-Poisson System, SIAM J. Numer. Anal. 39(2001), pp. 1146-1169. 
[12] F. Filbet, E. SonnendrüCKer, P. Bertrand, Conservative numerical schemes for the Vlasov equation, J. Comput. Phys. 172(2001), pp. 166-187.

[13] F. Filbet, E. SonnendrüCKer, Comparison of Eulerian Vlasov solvers, Comput. Phys. Comm. 151(2003), pp. 247-266.

[14] A. Ghizzo, P. Bertrand, M. Shoucri, T.W. Johnston, E. Fijalkow, M.R. FEIX, A Vlasov code for the numerical simulation of stimulated Raman scattering, J. Comput. Phys. 90(1990), pp. 431-457.

[15] F. Huot, A. Ghizzo, P. Bertrand, E. Sonnendrücker, O. Coulaud, Instability of the time splitting scheme for the one-dimensional and relativistic Vlasov-Maxwell equation, J. Comput. Phys. 185(2003), pp. 512-531.

[16] T.W. Johnston, P. Bertrand, A. Ghizzo, M. Shoucri, E. Fijalkow, M.R. FEIX, Simulated Raman scattering: Action evolution and particle trapping via Euler-Vlasov, fluid simulation, Phys. Fluids B4(1992), pp. 2523-2537.

[17] A.J. Klimas, W.M. Farrell, A splitting algorithm for Vlasov simulation with filamentation filtration, J. Comput. Phys. 110(1994), pp. 150-163.

[18] M. Shoucri, G. KnorR, Numerical integration of the Vlasov equation, J. Comput. Phys. 14(1974), pp. 84-92.

[19] E. Sonnendrücker, J. Roche, P. Bertrand, A. Ghizzo, The semiLagrangian method for the numerical resolution of the Vlasov equations, J. Comput. Phys. 149(1999), pp. 201-220. 